\title{
A Novel Mutation in the Thyroglobulin Gene Resulting in Neonatal Goiter and Congenital Hypothyroidism in an Eritrean Infant
}

\author{
(D) Eve Stern'1, (D) Nadia Schoenmakers², (D) Adeline K. Nicholas², (D) Eran Kassif3,4, (D) Orit Pinhas Hamiel1,4, (D) Yonatan Yeshayahu1,5 \\ ${ }^{1}$ Edmond and Lily Safra Children's Hospital, Sheba Medical Center, Pediatric Endocrine and Diabetes Unit, Ramat-Gan, Israel \\ ${ }^{2}$ Wellcome Trust-MRC Institute of Metabolic Science, University of Cambridge, Cambridge, United Kingdom \\ 3Sheba Medical Center, Department of Obstetrics and Gynecology, Tel Hashomer, Israel \\ ${ }^{4}$ Tel Aviv University, Sackler School of Medicine, Tel Aviv, Israel \\ 5 Faculty of Health Sciences, Ben-Gurion University, Beer-Sheva, Assuta Medical Center, Pediatric Endocrine and Diabetes Unit, Ashdod, Israel
}

\section{What is already known on this topic?}

Congenital hypothyroidism $(\mathrm{CH})$ may be caused by thyroid dysgenesis or thyroid dyshormonogenesis. Thyroid dyshormonogenesis is cause by mutations in genes involved in hormonogenesis and is usually inherited in an autosomal recessive manner. Mutations in the thyroglobulin gene $(T G)$ are a recognized cause of dyshormonogenic $\mathrm{CH}$, which may cause fetal or neonatal goiter

What this study adds?

The TG c.5686 + 1 delG pathogenic variant has not been previously described as causing congenital goitrous hypothyroidism.

\begin{abstract}
Congenital hypothyroidism $(\mathrm{CH})$ due to dyshormonogenesis may occur due to mutations in any of the key genes involved in thyroid hormone biosynthesis (TG, TPO, DUOX2, DUOXA2, SLC5A5, IYD, SLC26A4 and SLC26A7). Mutations in the thyroglobulin gene (TG) are frequently associated with goiter, which may present fetally or neonatally, although a spectrum of phenotypes is reported. We present the case of a woman of Eritrean origin who presented in the third trimester of pregnancy in the early stages of labor. Ultrasound at presentation revealed a fetal neck swelling consistent with a goiter. Following delivery by Caesarian section with minimal respiratory support, the infant was found to be hypothyroid with undetectable serum levels of thyroglobulin. Sequencing of the $T G$ revealed a homozygous donor splice site pathogenic variant (c.5686 + 1delG) not previously described in the literature. Levothyroxine treatment resulted in normal growth and psychomotor development. Goitrous $\mathrm{CH}$ with inappropriately low thyroglobulin has previously been reported in patients harbouring homozygous single nucleotide substitutions at the same $T G$ donor splice site, which result in exon skipping and retention of malformed thyroglobulin by the endoplasmic reticulum. We conclude that the TG c.5686+1delG pathogenic variant is the likely basis for our patient's fetal goiter and $\mathrm{CH}$, and that the clinical phenotype associated with TG c.5686 + 1 delG is comparable to that seen with single nucleotide substitutions at the same site.
\end{abstract}

Keywords: Congenital goiter, hypothyroidism, thyroglobulin, novel mutation, case report

Address for Correspondence: Eve Stern MD, Edmond and Lily Safra Children's Hospital, Sheba Medical Center, Pediatric Endocrine and Diabetes Unit, Ramat-Gan, Israel

Phone: +97235305015 E-mail: zipporaheve.stern@sheba.health.gov.il

ORCID: orcid.org/0000-0002-9716-3177

Presented in: This work was presented at the European Society of Pediatric Endocrinology annual meeting, Vienna, Austria, September 2019.

${ }^{\circ}$ Copyright 2022 by Turkish Society for Pediatric Endocrinology and Diabetes

The Journal of Clinical Research in Pediatric Endocrinology published by Galenos Publishing House.
Conflict of interest: None declared Received: 01.12 .2020 Accepted: 06.03.2021 


\section{Introduction}

Congenital hypothyroidism $(\mathrm{CH})$ is the commonest neonatal endocrine condition with recent reports demonstrating an incidence of around 1 in 1500 live births (1). Untreated $\mathrm{CH}$ can result in profound neurodevelopmental delay but since the introduction of newborn screening for $\mathrm{CH}$ in most Western countries, there has been a dramatic improvement in neurodevelopmental outcomes associated with the condition (2). $\mathrm{CH}$ is traditionally subdivided into thyroid dysgenesis (TD) and dyshormonogenesis, where TD refers to structural abnormalities of the thyroid including thyroid agenesis or an ectopic/sublingual thyroid gland and dyshormonogenesis describes inadequate thyroid hormone biosynthesis by a normally-located, often goitrous gland, due to molecular defects in the thyroid hormone biosynthetic machinery (3). Dyshormonogenesis usually has an identifiable monogenic basis, involving mutations in thyroglobulin $(T G)$ or genes involved in iodine transport, organification and recycling (SLC5A5, SLC26A4, DUOX2, DUOXA2, TPO, SLC26A7, IYD), although oligogenic mutations may also contribute (3). Inheritance of dyshormonogenesis is usually autosomal recessive although $\mathrm{CH}$ due to DUOX2, DUOXA2 and IYD mutations may also be dominantly inherited.

Dyshormonogenesis may present with goiter in the neonatal period or later, and may rarely be associated with fetal goiter. The mechanical consequences of fetal dyshormonogenic goiter may be associated with significant morbidity. Polyhydramnios may occur due to esophageal compression, and at delivery, neck hyperextension may result in malpresentation. Additionally, neonatal tracheal compression may cause fatal respiratory compromise. Complications can also arise from the underlying foetal thyroid dysfunction that can negatively affect development in utero and subsequent neurodevelopmental outcomes $(4,5,6,7)$.

The management of hypothyroid fetal goiter remains controversial. Since fetal goiter is a marker of serious thyroid dysfunction, many recommend the determination of thyroid function and the consideration of in utero treatment. Although fetal ultrasound, magnetic resonance imaging and amniotic fluid thyroid stimulating hormone (TSH) levels have all been methods suggested for determining fetal thyroid status, these have been shown to have variable levels of accuracy and cordocentesis has been recommended as the gold standard for determining fetal thyroid status. However, it must be taken into account that this may be associated with complications, including cord bleeding, chorioamnionitis and preterm delivery, among others (8).
When considering the decision to treat fetal goiter with associated hypothyroidism, one needs to take into account the size of the goiter, the effect on surrounding structures and associated features such as polyhydramnios. Treatment with both intra-amniotic levothyroxine and administration via the umbilical vein have been described with varying degrees of success, both with regards to reduction in goiter size, reduction in perinatal complications and improvement of fetal thyroid hormone levels $(5,6,7)$. However, conservative management, with radiological surveillance and elective delivery with respiratory support where necessary have also achieved favorable outcomes in some cases (4).

Genetic evaluation has been infrequently undertaken in cases with dyshormonogenic fetal goiter although underlying mutations in TG, TPO, and DUOXA2 have been reported in this context $(5,6,7)$.

\section{Case Report}

A 35-year-old woman of Eritrean origin was referred to a tertiary obstetric centre due to the finding of a neck mass on fetal ultrasound on presentation at term. Exact gestation was unknown as she had had no previous antenatal follow up or ultrasound scans.

Maternal history was notable for multiparity, with four previous healthy live births with no history of any congenital anomalies or $\mathrm{CH}$. She had no significant past medical history. Her non-consanguineous partner was also Eritrean with no significant past medical history.

On fetal ultrasound exam, a large mass was visualized in the neck and upper chest consisting of two lobes consistent with an enlarged thyroid gland (Figure 1). The mass including both lobes measured $55 \times 63 \mathrm{~mm}$. The trachea was noted to pass through the two lobes with no narrowing noted. Both carotids were displaced laterally by the mass. In addition, the superior vena cava was significantly enlarged, the heart was enlarged and significant tricuspid regurgitation was visualized.

In anticipation of difficulties in airway management following delivery, a Caesarean section was planned with pediatric ear nose and throat and anesthetic staff present. A live female infant was delivered in good condition with Apgar scores of 8 and 9 at one and five minutes respectively. Oxygen saturation was low and the infant was treated with high flow nasal cannula oxygen. Initial examination was notable for a large diffuse neck swelling (Figure 2) and mild respiratory distress with no other abnormal examination findings. The infant was transferred to the neonatal unit for further investigation and management. 
In light of prenatal ultrasound findings and clinical examination, initial studies were carried out to investigate thyroid structure and function. Initial thyroid function in the first 24 hours of life, showed primary hypothyroidism although free tri-iodothyronine (fT3) levels were preserved: TSH $272.4 \mathrm{mIU} / \mathrm{l}$ (0.4-20), free thyroxine (fT4) $6.3 \mathrm{pmol} / \mathrm{L}$ (10-30), and fT3 $5.5 \mathrm{pmol} / \mathrm{L}$ (2.5-9.8). Thyroglobulin was inappropriately low at $0.7 \mathrm{mcg} / \mathrm{L}(0-55)$, without detectable levels of thyroglobulin antibodies $(<20 \mathrm{U} / \mathrm{mL})$.

An ultrasound showed enlargement of both lobes of the thyroid gland, including the isthmus. The gland was reportedly of normal texture and was hyperemic. A thyroid technetium scan was also performed (Tc-99m) which demonstrated a diffusely enlarged thyroid gland with diffusely increased uptake.

In view of the laboratory findings of primary hypothyroidism, treatment was commenced on day 1 of life with high dose levothyroxine $(18 \mathrm{mcg} / \mathrm{kg})$ with rapid normalization of thyroid function tests. The dose was gradually tapered down accordingly. The child continues endocrine follow up and at the age of 3 years is currently well managed with

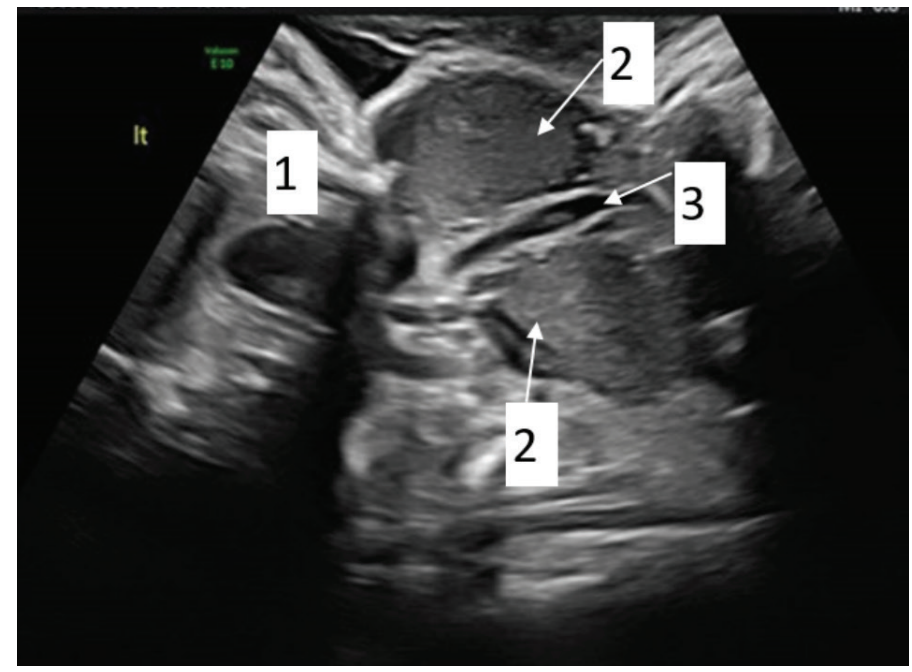

Figure 1. Foetal goiter as visualised on antenatal ultrasound. 1. Head, 2. Thyroid, 3. Trachea

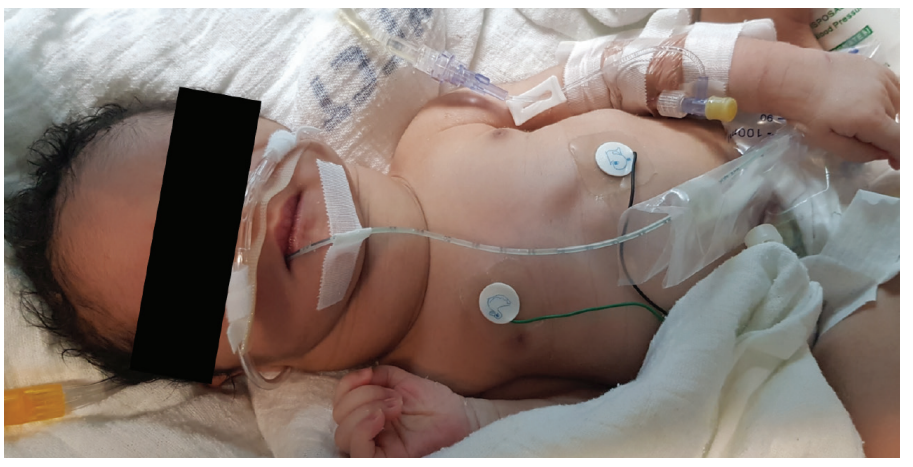

Figure 2. Neonatal goiter medical therapy, with a very small goiter which increases in size in accordance with increasing TSH levels. She has no additional medical problems and shows normal growth and psychomotor development.

Due to the association of neonatal goiter with laboratory evidence of hypothyroidism, low serum concentration of thyroglobulin and diffuse uptake of technetium on nuclear scanning, a genetic defect in the thyroglobulin gene was suspected and genetic studies were undertaken with written informed parental consent.

\section{Genetic Studies}

Sanger sequencing of the thyroglobulin gene (TG, ENST00000220616.9) revealed a novel donor splice site pathogenic variant at the exon 30-intron 30-31 boundary; c. $5686+1$ delG which is absent from the gnomAD database (Figure 3) (9). The patient was homozygous for this pathogenic variant. Due to parental reluctance to pursue further genetic testing, DNA was not available from her parents or four siblings for genotyping.

\section{Discussion}

Thyroglobulin is a large secretory protein which is crucial for thyroid hormone biosynthesis and storage in the thyroid follicular lumen. The $T G$ gene encodes a protein of 2768 amino acids in length including a 19 amino acid $\mathrm{N}$-terminal signal peptide. The recently-solved protein structure of TG has defined five regions ( $\mathrm{N}$-terminal domain, core, flap, arm and C-terminal domain) containing domains of type- 1 to type-3 cyteine rich TG repeats and a C-terminal cholineesterase-like domain (ChEL) as well as a probable four hormonogenic acceptor tyrosines and five donor tyrosines (10). TG is synthesized in the endoplasmic reticulum (ER) and folds with the assistance of molecular chaperones before trafficking to the apical membrane. The complex protein folding and intracellular trafficking of TG are essential for its normal follicular secretion and require both ER chaperones

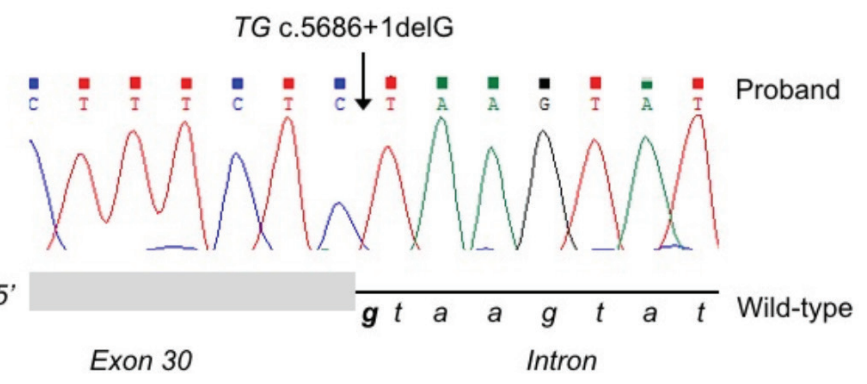

Figure 3. Sanger sequencing chromatogram for the $T G$ exon 30-intron boundary, demonstrating a single nucleotide deletion et the donor splice site (ENST00000220616.9, c.5686 + 1 delG)

TG: triglyceride gene 
and oxidoreductases, as well as specific intramolecular interactions (11). The ChEL domain has an important role in permitting TG intracellular trafficking and secretion and intradomain disulfide bonds between the many cysteine residues in TG are essential for the correct folding of newly synthesized TG $(11,12,13)$.

TG mutations are a common cause of dyshormonogenesis, with an estimated frequency of at least 1:100,000 and are usually inherited in an autosomal recessive manner, although $\mathrm{CH}$ has rarely been associated with monoallelic mutations $(13,14,15)$. To the best of our knowledge, $T G$ c. $5686+1$ delG has not previously been reported. However, three different point mutations resulting in single nucleotide substitutions at the same site have been reported in association with congenital goitrous hypothyroidism; TG c.5686+1G>A, $5686+1 G>C$ and $5686+1 G>T \quad(16,17,18,19,20,21)$. Analysis of patient-derived thyroidal tissue has confirmed that $T G$ c. $5686+1 \mathrm{G}>\mathrm{T}$ and $\mathrm{c} .5686+1 \mathrm{G}>\mathrm{C}$ mutations cause skipping of exon 30 with a resultant in-frame deletion of 46 amino acids in the TG type III repeat domain, causing the loss of 1 - putative N-linked glycosylation site and modifying the TG protein structure $(17,20)$. In common with most pathogenic $T G$ mutations, $T G$ c. $5686+1 \mathrm{G}>\mathrm{T}$ results in TG misfolding and retention within the ER with decreased export to the colloid (16). The fact that $T G$ c. $5686+1$ delG disrupts the same canonical donor splice site guanine residue, suggests that it is highly likely to be pathogenic, although functional studies to confirm this were not undertaken in our study. Due to parental reluctance to pursue further genetic testing, we were unable to obtain DNA to confirm segregation of homozygosity for the mutation with $\mathrm{CH}$ phenotype.

Individuals harbouring $T G$ mutations exhibit a spectrum of thyroid dysfunction, ranging from biochemically severe $\mathrm{CH}$ to euthyroid goiter (15). Goiter occurs frequently, commonly manifesting in the neonatal period, although onset may be delayed, and a small minority of cases exhibit fetal goiter $(6,18)$. The biochemical hallmark of $\mathrm{CH}$ due to a $T G$ mutation comprises an inappropriately low or undetectable circulating thyroglobulin level despite elevated circulating TSH concentration or goiter, and failure of exogenous TSH to stimulate a rise in serum TG. Thyroidal iodide uptake is enhanced and organification of iodide is usually preserved $(17,21)$. In some cases, the fT3/fT4 ratio is elevated (20).

Previously reported cases with homozygous disruption of the same donor splice site have shown variable biochemical phenotypes, although goiter is a consistent feature. Two Brazilian siblings who were homozygous for the TG c. $5686+1 \mathrm{G}>\mathrm{T}$ mutation exhibited fetal or neonatal goiter and severe $\mathrm{CH}$ (18). An additional two Brazilian siblings with the same mutation initially presented with congenital goiter. Interestingly, although the eldest sibling exhibited severe hypothyroidism, his sister had a milder biochemical phenotype with low serum total T4 but normal total T3. It was hypothesized that the variable expressivity seen in this family may be partly explained by iodine status, since the elder sibling was raised predominantly in an iodine deficient area whereas the family's relocation to an iodine replete region when his sister was aged 2 years may have ameliorated her thyroid dysfunction (17). A homozyous $T G$ c. $5686+1 \mathrm{G}>\mathrm{C}$ mutation was also identified in an iodine replete Pakistani girl, presenting aged 10 years with a massive goiter but normal TSH, subnormal fT4 and raised fT3 levels (20). It is likely that in this case and in the mildly hypothyroid Brazilian sibling, small quantities of mutated thyroglobulin molecules reached the follicular lumen, permitting iodination and synthesis of thyroid hormones, which is facilitated by adequate iodine intake. The elevated fT3/fT4 ratio may be at least in part due to increased thyroidal type 2 deiodinase activity (20). TG C $5686+1$ G > A was detected in compound heterozygosity with TG p.Q310P in two Japanese cases for whom detailed individual data was not presented although both were on treatment for screening-detected $\mathrm{CH}$ and had goiter in early childhood (21).

Here, we report a female patient of Eritrean origin with a large fetal goiter detected whilst in utero prior to delivery. Lack of ultrasound data from earlier in the pregnancy preclude definitive comments regarding the onset of goitrogenesis, however its large size and local compressive effects suggest it may have originated some weeks earlier. She had significant primary hypothyroidism at birth with preserved fT3 levels despite subnormal fT4 levels, and her thyroglobulin level was inappropriately low. These clinical and biochemical features are all recapitulated in previously reported patients harbouring mutations at the $T G$ c.5686 + 1 donor splice site and we believe her homozygous TG c. $5686+1$ delG pathogenic variant to be the likely cause of her thyroid dysfunction, although future functional studies will be required to confirm this.

Urinary iodine was not measured contemporaneously with presentation in this patient but since there is evidence to suggest that iodine intake during pregnancy may be inadequate in some areas of Israel, suboptimal maternal iodine status may have contributed to the fetal goitrogenesis although the patient's normal fT3 suggests some iodination of mutant TG was occurring in the follicular lumen. Although conservative management of her fetal goiter resulted in a relatively uncomplicated delivery, radiological monitoring for fetal goiter would be advisable during future pregnancies, 
given the presumed $25 \%$ risk of having another affected child if both parents are heterozygotes for the $T G$ c.5686+1 delG pathogenic variant. Additionally, maternal iodine status should be optimized.

\section{Conclusion}

We report a case of an infant presenting in late pregnancy with a large fetal goiter, $\mathrm{CH}$ determined by laboratory studies following delivery and an undetectable serum thyroglobulin. Sequencing of the thyroglobulin gene revealed an as yet unreported homozygous donor splice site pathogenic variant at the exon 30-intron 30-31 boundary; c. $5686+1$ delG. Previous single nucleotide substitutions and this site have been described with variable phenotypes including both neonatal goiter, goitrous hypothyroidism, and goitrous euthyroidism. Genetic evaluation, when carried out, revealed skipping of exon 30 in the mRNA and subsequent generation of a shortened protein. Functional evaluation of single nucleotide substitutions at this site demonstrated retention of the TG protein within the ER and resulting decreased export to the follicular lumen. Due to parental refusal further genetic testing on unaffected family members was not carried out. However, based on previous studies as described and the patient's clinical, biochemical and imaging phenotype, we concluded that this pathogenic variant was the likely cause for the patient's clinical condition.

\section{Acknowledgements}

The authors wish to thank Mrs. Carmel Byers for her assistance in acquiring genetic testing.

\section{Ethics}

Informed Consent: Consent form was filled out by all participants.

Peer-review: Externally peer-reviewed.

\section{Authorship Contributions}

Surgical and Medical Practices: Adeline K. Nicholas, Concept: Eve Stern, Eran Kassif, Orit Pinhas Hamiel, Design: Eve Stern, Eran Kassif, Orit Pinhas Hamiel, Nadia Schoenmakers Data Collection or Processing: Nadia Schoenmakers, Analysis or Interpretation: Eve Stern, Nadia Schoenmakers, Adeline K. Nicholas, Eran Kassif, Orit Pinhas Hamiel, Yonatan Yeshayahu, Literature Search: Eve Stern, Nadia Schoenmakers, Adeline K. Nicholas, Eran Kassif, Orit Pinhas Hamiel, Yonatan Yeshayahu, Writing: Eve Stern, Nadia Schoenmakers, Adeline K. Nicholas, Eran Kassif, Orit Pinhas Hamiel, Yonatan Yeshayahu.
Financial Disclosure: The authors declared that this study received no financial support.

\section{References}

1. Corbetta C, Weber G, Cortinovis F, Calebiro D, Passoni A, Vigone MC, Beck-Peccoz P, Chiumello G, Persani L. A 7-year experience with low blood TSH cutoff levels for neonatal screening reveals an unsuspected frequency of congenital hypothyroidism $(\mathrm{CH})$. Clin Endocrinol (Oxf) 2009;71:739-745. Epub 2009 Mar 28

2. Grosse SD, Van Vliet G. Prevention of intellectual disability through screening for congenital hypothyroidism: how much and at what level? Arch Dis Child 2011;96:374-379. Epub 2011 Jan 17

3. Peters C, van Trotsenburg ASP, Schoenmakers N. DIAGNOSIS OF ENDOCRINE DISEASE: Congenital hypothyroidism: update and perspectives. Eur J Endocrinol 2018;179:297-317.

4. Reynolds BC, Simpson JH, Macara L, Watt AJ, Kubba H, Donaldson MD, Pohlen J. Goitrous congenital hypothyroidism in a twin pregnancy causing respiratory obstruction at birth: implications for management. Acta Paediatr 2006;95:1345-1348.

5. Börgel K, Pohlenz J, Holzgreve W, Bramswig JH. Intrauterine therapy of goitrous hypothyroidism in a boy with a new compound heterozygous mutation (Y453D and C800R) in the thyroid peroxidase gene. A longterm follow-up. Am J Obstet Gynecol 2005;193:857-858.

6. Vasudevan P, Powell C, Nicholas AK, Scudamore I, Greening J, Park $\mathrm{SM}$, Schoenmakers $\mathrm{N}$. Intrauterine death following intraamniotic triiodothyronine and thyroxine therapy for fetal goitrous hypothyroidism associated with polyhydramnios and caused by a thyroglobulin mutation. Endocrinol Diabetes Metab Case Rep 2017;2017:17-0040.

7. Tanase-Nakao K, Miyata I, Terauchi A, Saito M, Wada S, Hasegawa T, Narumi S. Fetal Goitrous Hypothyroidism and Polyhydramnios in a Patient with Compound Heterozygous DUOXA2 Mutations. Horm Res Paediatr 2018;90:132-137. Epub 2018 Aug 15

8. Iijima S. Current knowledge about the in utero and peripartum management of fetal goiter associated with maternal Graves' disease. Eur J Obstet Gynecol Reprod Biol X 2019;3:100027.

9. Karczewski KJ, Francioli LC, Tiao G, Cummings BB, Alföldi J, Wang Q, Collins RL, Laricchia KM, Ganna A, Birnbaum DP, Gauthier LD, Brand H, Solomonson M, Watts NA, Rhodes D, Singer-Berk M, England EM, Seaby EG, Kosmicki JA, Walters RK, Tashman K, Farjoun Y, Banks E, Poterba T, Wang A, Seed C, Whiffin N, Chong JX, Samocha KE, PierceHoffman E, Zappala Z, O’Donnell-Luria AH, Minikel EV, Weisburd B, Lek M, Ware JS, Vittal C, Armean IM, Bergelson L, Cibulskis K, Connolly KM, Covarrubias M, Donnelly S, Ferriera S, Gabriel S, Gentry J, Gupta N, Jeandet T, Kaplan D, Llanwarne C, Munshi R, Novod S, Petrillo N, Roazen D, Ruano-Rubio V, Saltzman A, Schleicher M, Soto J, Tibbetts K, Tolonen C, Wade G, Talkowski ME; Genome Aggregation Database Consortium, Neale BM, Daly MJ, MacArthur DG. The mutational constraint spectrum quantified from variation in 141,456 humans. Nature. 2020;581:434-443. doi: 10.1038/s41586-020-2308-7. Epub 2020 May 27. Erratum in: Nature 2021;590:E53. Erratum in: Nature. 2021;597:E3-E4. PMID: 32461654; PMCID: PMC7334197.

10. Coscia F, Taler-Verčič A, Chang VT, Sinn L, O’Reilly FJ, Izoré T, Renko M, Berger I, Rappsilber J, Turk D, Löwe J. The structure of human thyroglobulin". Nature 2020;578:627-630. Epub 2020 Feb 5.

11. Di Jeso B, Arvan P. Thyroglobulin From Molecular and Cellular Biology to Clinical Endocrinology. Endocr Rev 2016;37:2-36. Epub 2015 Nov 23

12. Lee J, Di Jeso B, Arvan P. The cholinesterase-like domain of thyroglobulin functions as an intramolecular chaperone. J Clin Invest 2008;11 8:29502958. 
13. Citterio CE, Targovnik HM, Arvan P. The role of thyroglobulin in thyroid hormonogenesis. Nat Rev Endocrinol 2019;15:323-338.

14. Siffo S, Adrover E, Citterio CE, Miras MB, Balbi VA, Chiesa A, Weill J, Sobrero G, González VG, Papendieck P, Martinez EB, Gonzalez-Sarmiento R, Rivolta CM, Targovnik HM. Molecular analysis of thyroglobulin mutations found in patients with goiter and hypothyroidism. Mol Cell Endocrinol 2018;473:1-16. Epub 2017 Dec 22

15. Targovnik HM, Citterio CE, Rivolta CM. Iodide handling disorders (NIS, TPO, TG, IYD). Best Pract Res Clin Endocrinol Metab 2017;31:195-212. Epub 2017 Apr 4.

16. Medeiros-Neto G, Kim PS, Yoo SE, Vono J, Targovnik HM, Camargo R, Hossain SA, Arvan P. Congenital hypothyroid goiter with deficient thyroglobulin. Identification of an endoplasmic reticulum storage disease with induction of molecular chaperones. J Clin Invest 1996;98:2838-2844.

17. Targovnik HM, Rivolta CM, Mendive FM, Moya CM, Vono J, MedeirosNeto G. Congenital goiter with hypothyroidism caused by a 5' splice site mutation in the thyroglobulin gene. Tyroid 2001;11:685-690.

18. Pardo V, Rubio IG, Knobel M, Aguiar-Oliveira MH, Santos MM, Gomes SA, Oliveira CR, Targovnik HM, Medeiros-Neto G. Phenotypic variation among four family members with congenital hypothyroidism caused by two distinct thyroglobulin gene mutations. Thyroid 2008;18:783786.

19. Pardo V, Vono-Toniolo J, Rubio IG, Knobel M, Possato RF, Targovnik HM, Kopp P, Medeiros-Neto G. The p.A2215D thyroglobulin gene mutation leads to deficient synthesis and secretion of the mutated protein and congenital hypothyroidism with wide phenotype variation. J Clin Endocrinol Metab 2009;94:2938-2944. Epub 2009 Jun 9

20. Hermanns P, Refetoff S, Sriphrapradang C, Pohlenz J, Okamato J, Slyper L, Slyper AH. A clinically euthyroid child with a large goiter due to a thyroglobulin gene defect: clinical features and genetic studies. J Pediatr Endocrinol Metab 2013;26:119-123.

21. Hishinuma A, Fukata S, Nishiyama S, Nishi Y, Oh-Ishi M, Murata Y, Ohyama Y, Matsuura N, Kasai K, Harada S, Kitanaka S, Takamatsu J, Kiwaki K, Ohye H, Uruno T, Tomoda C, Tajima T, Kuma K, Miyauchi A, Ieiri T. Haplotype analysis reveals founder effects of thyroglobulin gene mutations C1058R and C1977S in Japan. J Clin Endocrinol Metab 2006;91:3100-3104. 